\title{
Continuity Enhancement Method for Real-Time PPP Based on Zero-Baseline Constraint of Multi-Receiver
}

\author{
Fuxin Yang $\mathbb{D}^{\text {, }}$ Chuanlei Zheng, Hui Li *, Liang Li, Jie Zhang and Lin Zhao
}

Citation: Yang, F.; Zheng, C.; Li, H.; Li, L.; Zhang, J.; Zhao, L. Continuity Enhancement Method for Real-Time PPP Based on Zero-Baseline Constraint of Multi-Receiver. Remote Sens. 2021, 13, 605. https://doi.org/ $10.3390 /$ rs13040605

Academic Editor: José Fernández Received: 20 December 2020

Accepted: 5 February 2021

Published: 8 February 2021

Publisher's Note: MDPI stays neutral with regard to jurisdictional claims in published maps and institutional affiliations.

Copyright: (c) 2021 by the authors. Licensee MDPI, Basel, Switzerland. This article is an open access article distributed under the terms and conditions of the Creative Commons Attribution (CC BY) license (https:/ / creativecommons.org/licenses/by/ $4.0 /)$.
College of Intelligent Systems Science and Engineering, Harbin Engineering University, Harbin 150001, China; yangfuxin@hrbeu.edu.cn (F.Y.); zhcl@hrbeu.edu.cn (C.Z.); liliang@hrbeu.edu.cn (L.L.); jiezhang@hrbeu.edu.cn (J.Z.); zhaolin@hrbeu.edu.cn (L.Z.)

* Correspondence: lihuiheu@hrbeu.edu.cn; Tel.: +86-451-8256-8587

\begin{abstract}
Continuity is one of the metrics that characterize the required navigation performance of global navigation satellite system (GNSS)-based applications. Data outage due to receiver failure is one of the reasons for continuity loss. Although a multi-receiver configuration can maintain position solutions in case a receiver has data outage, the initialization of the receiver will also cause continuous high-precision positioning performance loss. To maintain continuous high-precision positioning performance of real-time precise point positioning (RT-PPP), we proposed a continuity enhancement method for RT-PPP based on zero-baseline constraint of multi-receiver. On the one hand, the mean time to repair (MTTR) of the multi-receiver configuration is improved to maintain continuous position solutions. On the other hand, the zero-baseline constraint of multi-receiver including between-satellite single-differenced (BSSD) ambiguities, zenith troposphere wet delay (ZWD), and their suitable stochastic models are constructed to achieve instantaneous initialization of back-up receiver. Through static and kinematic experiments based on real data, the effectiveness and robustness of proposed method are evaluated comprehensively. The experiment results show that the relationship including BSSD ambiguities and ZWD between receivers can be determined reliably based on zero-baseline constraint, and the instantaneous initialization can be achieved without high-precision positioning continuity loss in the multi-receiver RT-PPP processing.
\end{abstract}

Keywords: continuity; real-time precise point positioning; multi-receiver; instantaneous initialization

\section{Introduction}

Precise point positioning (PPP) has advantages of high computational efficiency and flexible operating mode without requiring dedicated reference stations, which is a desired precise positioning method for mass-market applications [1,2]. Until recently, some commercial companies including Fugro, Veripos, and Trimble provided real-time precise point positioning (RT-PPP) service in marine and agriculture areas. Although the RT-PPP can provide high-precision position solution, it requires a relatively long initialization period to obtain position accuracy of dm-level or even cm-level [3,4]. To ensure that RT-PPP meets the requirements of real-time applications in maritime, air, and even automotive navigation, there is a growing interest in instant and continuous precision solutions of RT-PPP.

The first initialization and re-initialization events are the main factors affecting instant and continuous precision solutions of RT-PPP. RT-PPP takes tens of minutes for the phase ambiguity to converge to the desired accuracy of centimeter-level, and the initialization time depends on satellite geometry and prevailing atmospheric conditions [5,6]. With the assistance of the uncalibrated phase delay (UPD) products and some atmospheric products such as tropospheric and ionospheric delay corrections, the PPP-RTK (real-time kinematic) method was proposed to achieve position accuracy at the centimeter-level with an initialization time of $1 \mathrm{~min}$ or better [7-9]. In addition, we can also spend tens of minutes waiting for RT-PPP to complete the first initialization. Therefore, the instant and continuous precision 
solutions of RT-PPP are mainly limited by the re-initialization due to corrections outage, cycle slips, receiver data outage, and so on. In terms of instant and continuous RT-PPP, scholars have made some achievements. El-Mowafy proposed the prediction method of real-time orbit and clock corrections to maintain precision solutions in case the communication link experiences outages $[10,11]$. To overcome the re-initialization, a number of cycle slip detection and correction methods have been developed and implemented to achieve instantaneous re-initialization of PPP, even if the ionosphere index is active [12-15]. However, the continuity loss caused by single receiver data outage (raw observation loss) is often ignored, and the re-initialization of RT-PPP happens due to the cycle slip repair failure with a long data outage.

In order to avoid loss of positioning continuity caused by single receiver failure, a multiple receiver configuration is always applied in order to enhance the reliability of precise positioning in navigation, geodesy, land surveying, and geo-dynamics [16,17]. Although an Inertial Measurement Unit (IMU) would effectively bridge-gap the receiver outage in a fused sensor implementation, the continuous high-precision positioning performance will be lost due to a single IMU sensor for a long time [18]. The survey vessels usually set up independent receivers with PPP function at different positions to back up each other for continuous high-precision positioning, such as dynamic positioning system and unmanned vehicle. The definition of receiver failure is the time period when the receiver fails and the raw observation cannot be received to resolve position solutions. Although the configuration strategy of multiple receivers sharing the same antenna can be used to meet the continuity requirements, the initialization of the back-up receiver will also destroy the continuous high-precision positioning performance in case the master receiver has a data outage. Considering the rapid initialization of ambiguity is the essence of the RT-PPP initialization, thus, Song and Hao developed an instantaneous re-initialization method by the use of relationship between multiple receiver ambiguities for PPP [17]. They focus on instant precision positioning, which will cause instantaneous re-initialization failure with constant baseline constraint by ignoring baseline deformation. The success rate of doubledifferences ambiguity fixing is $99 \%$, and the method is verified with limited experimental data. Meanwhile, recent research has shown that the reliability of instantaneous initialization can be improved by introducing external troposphere constraints and establishing proper stochastic model [19-21]. Furthermore, there are few studies regarding instant and continuous precision positioning of RT-PPP in multi-receiver strategy, especially to improve the continuous high-precision positioning performance.

In this contribution, in order to meet the requirements of applications with reliability of high-precision positioning and avoid continuous loss even if the frequent data outages occur within a few minutes in both master and back-up receivers, such as dynamic positioning system and unmanned vehicle, a new method is proposed to enhance continuous high-precision positioning performance of RT-PPP based on zero-baseline constraint of multi-receiver. Firstly, a dual frequency between-satellite single-difference (BSSD) ionosphere-free (IF) PPP model is derived, and the float states are calculated based on robust sequential least squares (RSLQS). Secondly, compared to non-zero fixed baseline constraint using dual-antenna, the issue of double difference ambiguity fixing reliability reduction due to dual-antenna baseline deformation can be avoided with zero-baseline constraint using a single antenna, and the independently single antenna with multiple receiver configuration can be set up at different positions to back up each other to avoid antenna failure. The benefit from zero baseline constraint is that not only the relationship of between-satellite single-difference ambiguities between receivers is established, but also the zenith wet tropospheric constraint between receivers is established as additional observation to improve the model strength. Meanwhile, in accordance to the law of error propagation, a suitable stochastic model is established to assign the appropriate weights for the constraints. Therefore, the raw measurements of back-up receiver with prior information of previous epoch's zero-baseline constraints can achieve instant initialization during the master receiver's observation outage. In addition, the re-initialization in a receiver 
caused by cycle slips can be handled by [15] to achieve instant high-precision positioning. In the experiment section, we present results from static and kinematic data to assess the performance of this new method. Finally, some conclusions and remarks are summarized.

\section{Methodology}

The raw observations from station $i(I=1,2, \ldots, m, s)$ to satellite $j(j=1,2, \ldots, l, k)$ can be simplified as [22]:

$$
\begin{gathered}
L_{i, g}^{j}=\rho_{i}^{j}+d t_{i}-d t^{j}-\gamma_{i, g} \cdot I_{i, 1}^{j}+T_{i}^{j}+\lambda_{g} \cdot\left(N_{i, g}^{j}+b_{i, g}^{j}\right)+\xi_{i, g}^{j} \\
P_{i, g}^{j}=\rho_{i}^{j}+d t_{i}-d t^{j}+\gamma_{i, g} \cdot I_{1}^{j}+T_{i}^{j}+d_{i, g}^{j}+\varepsilon_{i, g}^{j}
\end{gathered}
$$

where $L$ and $P$ denote carrier phase and code observations, respectively; $g(g=1,2)$ denotes observation frequency, L1/L2 for GPS and GLONASS, B1/B2 for BDS, and E1/E5a for Galileo. $\rho_{i}^{j}$ denotes the geometric distance between satellite $j$ and rover $i, d t_{i}$ and $d t^{j}$ are the clock biases of receiver and satellite, respectively; $I_{i, 1}^{j}$ is the slant ionosphere refraction on $f_{1}, \gamma_{g}=f_{1}^{2} / f_{g}^{2}$ is the ionospheric factor of $f_{g}$ with respect to $f_{1} ; T_{i}^{j}$ is slant tropospheric delay; $\lambda_{g}$ is the carrier phase wavelength at $f_{g} ; N_{i, g}^{j}$ is the integer phase ambiguity at $f_{g}$; $d_{i, g}^{j}=d_{i, g}-d_{g}^{j}$ is the difference of code biases between receiver and satellite; $b_{i, g}^{j}=b_{i, g}-b_{g}^{j}$ is the difference of uncalibrated phase delays (UPDs) between receiver and satellite. $\varepsilon_{i, g}^{j}$ and $\xi_{i, g}^{j}$ are the sum of multipath effects and thermal noise for the code and carrier phase observations, respectively. Note that all variables are expressed in meters, except the ambiguity and UPDs which are in cycles. In addition, the relativity effects, the phase wind-up, and the site displacement effects, including solid earth tides, ocean tides and polar tides, are corrected through the empirical models $[23,24]$.

As is well-known, the IF combination observations are normally used in PPP to eliminate the first-order ionospheric delays in the pseudo-range and carrier phase measurements, which can be expressed as follows [25],

$$
\begin{gathered}
L_{i, I F}^{j}=\alpha L_{i, 1}^{j}+(1-\alpha) L_{i, 2}^{j}=\rho_{i}^{j}+d t_{i}+T_{i}^{j}+\lambda_{I F} \widetilde{N}_{i, I F}^{j}+\tilde{\zeta}_{i, I F}^{j} \\
P_{i, I F}^{j}=\alpha P_{i, 1}^{j}+(1-\alpha) P_{i, 2}^{j}=\rho_{i}^{j}+d t_{i}+d_{i, I F}+T_{i}^{j}+\varepsilon_{i, I F}^{j}
\end{gathered}
$$

where $\alpha=f_{1}^{2} /\left(f_{1}^{2}-f_{2}^{2}\right), \lambda_{I F} \widetilde{N}_{i, I F}^{j}=\alpha\left(\lambda_{1} N_{i, 1}^{j}+b_{i, 1}^{j}\right)+(1-\alpha)\left(\lambda_{2} N_{i, 2}^{j}+b_{i, 2}^{j}\right)$ is the IF ambiguity in meters, $d_{i, I F}=\alpha \cdot d_{i, 1}+(1-\alpha) \cdot d_{i, 2}$ is the IF receiver code hardware delays in meters. The satellite orbit and clock can be corrected using external precise products in state space representation (SSR) format. It should be noted that the current real-time precise clock products are referred to the ionospheric-free combination of L1/L2 for GPS, E1/E5a for Galileo, and B1/B2 for BDS. As a result, there is no satellite code bias on the right side of (4) for the IF combination which is adopted to generate the precise clock products. For the carrier phase, the uncalibrated phase delays cannot be cancelled and will be mapped into ambiguities. This mapping will not be a problem since the ambiguities are estimated as a lumped term and treated as float values [26].

\subsection{BSSD Ambiguity-Float PPP Model}

The BSSD observations are employed in this section, so that the effects of receiver clock error as well as receiver-dependent code and phase hardware delays in (3) and (4) can be removed. The IF PPP model based on BSSD for receiver $m$ between satellites $l$ and $k$ can be expressed as,

$$
\Delta L_{m, I F}^{l k}=\Delta \rho_{m}^{l k}+m f(e l)_{m, w}^{l k} Z_{m, w}+\lambda_{I F} \Delta \tilde{N}_{m, I F}^{l k}+\Delta \xi_{m, I F}^{l k}
$$




$$
\Delta P_{m, I F}^{l k}=\Delta \rho_{m}^{l k}+m f(e l)_{m, w}^{l k} Z_{m, w}+\Delta \varepsilon_{m, I F}^{l k}
$$

where $\Delta$ denotes the satellite-between differenced, and the satellite $k$ with healthy observations and the highest elevation is selected to the reference. $e l$ denotes the elevation. $Z_{m, w}$ is zenith wet delay (ZWD) of troposphere, which is estimated based on the $2 \mathrm{~h}$ piecewise method. $m f(e l)_{m, w}$ denotes the non-hydrostatic mapping functions at $e l$ for station $m$ [27]. Therefore, the estimated states contain position, ZWD, and BSSD float carrier phase ambiguities of all satellites in view for station $m$. Specifically, the PPP algorithm can be implemented using RSLQS [1]. The float-estimated states by RSLQS can be obtained as follows,

$$
\begin{gathered}
\hat{\boldsymbol{x}}_{m}=\left(\boldsymbol{P}_{m, 0}+\boldsymbol{H}_{m}^{T} \boldsymbol{R}_{m}^{-1} \boldsymbol{H}_{m}\right)^{-1} \boldsymbol{H}_{m}^{T} \boldsymbol{R}_{m}^{-1} \boldsymbol{Y}_{m}+\left[\begin{array}{lll}
\hat{\boldsymbol{a}}_{m, 0} & \hat{\boldsymbol{b}}_{m, 0} & \hat{\boldsymbol{c}}_{m, 0}
\end{array}\right]^{T}=\left[\begin{array}{lll}
\hat{\boldsymbol{a}}_{m} & \hat{\boldsymbol{b}}_{m} & \hat{\boldsymbol{c}}_{m}
\end{array}\right]^{T} \\
\boldsymbol{Q}_{m, \boldsymbol{x}}=\left(\boldsymbol{P}_{m, 0}+\boldsymbol{H}_{m}^{T} \boldsymbol{R}_{m}^{-1} \boldsymbol{H}_{m}\right)^{-1}=\left[\begin{array}{ccc}
\boldsymbol{Q}_{m, a} & \boldsymbol{Q}_{m, a b} & \boldsymbol{Q}_{m, a c} \\
\boldsymbol{Q}_{m, b a} & \boldsymbol{Q}_{m, b} & \boldsymbol{Q}_{m, b c} \\
\boldsymbol{Q}_{m, c a} & \boldsymbol{Q}_{m, c b} & \boldsymbol{Q}_{m, c}
\end{array}\right]
\end{gathered}
$$

where $\hat{x}_{m}$ is the BSSD float states with variance-covariance matrix (VC-matrix) $Q_{m, x}, \hat{\boldsymbol{a}}_{m}$, $\hat{\boldsymbol{b}}_{m}$, and $\hat{\boldsymbol{c}}_{m}$ denote the position coordinates $(X, Y, Z), Z W D$, and BSSD float carrier phase ambiguities of all satellites in view, respectively. The subscript 0 denotes prior value. $\boldsymbol{H}_{m}$ is the design matrix, $\boldsymbol{P}_{m, 0}$ is a priori weighted matrix for the float-estimated states, $\boldsymbol{R}_{m}$ is the robust observation weight matrix, and $\boldsymbol{Y}_{m}$ is the corrected observation vector.

\subsection{Zero Baseline Constraint Conditions}

The multi-receiver with zero-baseline constraint usually contains master receiver $m$ and back-up receiver $s$. In order to achieve instantaneous initialization of back-up receiver $s$ when raw data outage occurs in the receiver $m$, the states of receiver $s$ contain BSSD IF float carrier phase ambiguities, ZWD and their stochastic model should be determined precisely.

On the one hand, the double-differenced ambiguities $\nabla \Delta \boldsymbol{N}_{m s, 1}^{k l}$ and $\nabla \Delta \boldsymbol{N}_{m s, 2}^{k l}$ can be fixed using the least-squares ambiguity decorrelation adjustment (LAMBDA) algorithm [28]. Meanwhile, the ambiguities relationship between master receiver $m$ and back-up receiver $s$ can be determined reliably by double-differenced model with zero baseline constraints [29]. The constraint condition is that $\sqrt{\left(X_{m}-X_{b}\right)^{2}+\left(Y_{m}-Y_{b}\right)^{2}+\left(Z_{m}-Z_{b}\right)^{2}}=0$, where $\left(X_{m}, Y_{m}, Z_{m}\right)$ and $\left(X_{b}, Y_{b}, Z_{b}\right)$ are master and back-up positions in the Earth Centered Earth Fixed (ECEF) coordinate system, respectively. Once the double-differenced ambiguities $\nabla \Delta N_{m s, 1}^{k l}$ and $\nabla \Delta N_{m s, 2}^{k l}$ are fixed separately, the prior BSSD float carrier phase ambiguities of receiver $s$ can be written as follows,

$$
c_{s, 0}=\hat{c}_{m}-\hat{c}_{m s}
$$

where $\hat{\boldsymbol{c}}_{m s}=\alpha \cdot \nabla \Delta \boldsymbol{N}_{m s, 1}^{k l}+(1-\alpha) \cdot \nabla \Delta \boldsymbol{N}_{m s, 2}^{k l}$ denotes the double-differenced IF ambiguities between master receiver $m$ and back-up receiver $s$. On the other hand, the prior ZWD of back-up receiver $s$ with zero baseline constraints can be written as,

$$
\boldsymbol{b}_{s, 0}=\hat{\boldsymbol{b}}_{m}
$$

Therefore, the zero baseline constraint conditions including BSSD IF ambiguities and ZWD can be constructed reliably based on BSSD ambiguity-float PPP model.

Inappropriate weights may cause outliers to remain undetected and truly high-quality observations to be rejected, thus leading to a considerable loss of accuracy despite largely redundant observations. Since $\hat{\boldsymbol{c}}_{m s}$ can be expressed as $\hat{\boldsymbol{c}}_{m s}=\alpha \cdot \nabla \Delta \boldsymbol{N}_{m s, 1}^{k l}+(1-\alpha) \cdot \nabla \Delta \boldsymbol{N}_{m s, 2}^{k l}$ $\hat{c}_{m s}$ become a constant value when the double-differenced ambiguities $\nabla \Delta N_{m s, 1}^{k l}$ and 
$\nabla \Delta \boldsymbol{N}_{m s, 2}^{k l}$ are fixed. Therefore, the variance of $\hat{\boldsymbol{c}}_{m s}$ is zero. In accordance with the variancecovariance propagation law, the prior VC-matrix of (9) and (10) can be expressed as follows,

$$
\begin{gathered}
\boldsymbol{Q}_{s, c 0}=D\left\{\boldsymbol{c}_{s, 0}\right\}=D\left\{\hat{\boldsymbol{c}}_{m}\right\}=\boldsymbol{Q}_{m, c} \\
\boldsymbol{Q}_{s, b 0}=D\left\{\boldsymbol{b}_{s, 0}\right\}=D\left\{\hat{\boldsymbol{b}}_{m}\right\}=\boldsymbol{Q}_{m, b}
\end{gathered}
$$

where, $D\{\cdot\}$ denotes variance operator. Meanwhile, $\operatorname{Cov}\left(\boldsymbol{b}_{s, 0}, \boldsymbol{c}_{s, 0}\right)=\operatorname{Cov}\left(\hat{\boldsymbol{b}}_{m}, \hat{\boldsymbol{c}}_{m}\right)=\boldsymbol{Q}_{m, b c}$ and $\operatorname{Cov}\left(\boldsymbol{c}_{s, 0}, \boldsymbol{b}_{s, 0}\right)=\boldsymbol{Q}_{m, c b}$, Therefore, the suitable stochastic model to assign the appropriate weights for zero baseline constraint conditions can be obtained.

\subsection{Instantaneous Initialization with Zero-Baseline Constraint of the Back-Up Receiver}

Once the raw data outage occurs in the receiver $m$, the prior BSSD IF ambiguities and ZWD can be determined by (9) and (10), and their VC-matrix can be obtained based on (11) and (12). The prior VC-matrix of receiver $s$ float-estimated states can be written as follows,

$$
\boldsymbol{P}_{s, 0}=\left[\begin{array}{ccc}
\boldsymbol{Q}_{s, a} & \mathbf{0} & \mathbf{0} \\
\mathbf{0} & \boldsymbol{Q}_{m, b} & \boldsymbol{Q}_{m, b c} \\
\mathbf{0} & \boldsymbol{Q}_{m, c b} & \boldsymbol{Q}_{m, c}
\end{array}\right]
$$

where $Q_{s, a}$ is a priori VC-matrix for $\hat{\boldsymbol{a}}_{s, 0} . \hat{\boldsymbol{a}}_{s, 0}$ can be calculated by weighted least squares. Furthermore, the float-estimated states of receiver $s$ can be calculated based on (7) and (13). Therefore, the continuity loss of high-precision position solutions due to a single receiver data outage can be avoided. The PPP engine calculates each epoch's solution based on back-up receiver until the outage occurs in the back-up receiver.

The benefits of proposed method are concluded according to the description mentioned above. On the one hand, the benefit from zero baseline constraints, the issue of double difference (DD) ambiguity fixing reliability reduction due to dual-antenna baseline deformation can be avoid. Meanwhile, in accordance with the law of error propagation, the BSSD IF ambiguities and ZWD of back-up receiver calculated based on zero-baseline constraint have the same accuracy as the master receiver, and the initialization of BSSD IF phase ambiguity and ZWD can be achieved instantly when the master receiver experiences data outage. The appropriate stochastic model is also established to match the zero-baseline constraints. Therefore, the ZWD and BSSD float carrier phase ambiguities of all satellites in view for the back-up receiver can achieve instant initialization, and the instantaneous initialization or re-initialization can be achieved. On the other hand, compared to a single receiver, the mean time to repair (MTTR) of multi-receiver configuration is improved to ensure the continuity of position solutions, even if frequent outages occur to both receivers. Therefore, the proposed method can meet the requirements of applications with reliability of high-precision positioning and avoid continuous loss due to a single receiver failure. MTTR indicates the time length to achieve high-precision positioning after a raw data outage occurs in a single receiver.

The flow chart of the proposed method is shown in Figure 1.

Step A: When the master and back-up receiver operate normally sharing the same antenna, the PPP engine calculates each epoch's solution based on master receiver's measurements, and the back-up receiver just receives raw data. Meanwhile, the zerobaseline constraints including BSSD IF ambiguities, ZWD, and their VC-matrixes are calculated in parallel all the time. The BSSD IF ambiguities and ZWD of back-up receiver can be calculated based on (9) and (10) at each epoch, and the VC-matrix of constraints can also be obtained based on (11) and (12). In accordance to the law of error propagation, the BSSD IF ambiguities and ZWD of back-up receiver have the same accuracy as the master. Although the PPP solution can be calculated from both receivers all the time, and switch to one of them if the other fails, the continuous high-precision positioning performance will be lost due to re-initialization when frequent data outages occur within a few minutes in both master and back-up receivers. 
Step B: Once the master receiver's observation outage occurs, the system switches to the back-up receiver's raw measurements. The zero-baseline constraints including BSSD IF ambiguities, ZWD, and their VC-matrixes as the prior information of back-up receiver is determined at previous epoch or earlier to instantly achieve initialization by (7), and the continuity loss can be avoided by instantaneous initialization. Note that the re-initialization in a receiver caused by cycle slips can be handled by [15] to achieve instant high-precision positioning. During the master receiver's observation outage, the PPP engine calculates each epoch's solution based on back-up receiver until the outage occurs in the back-up receiver.

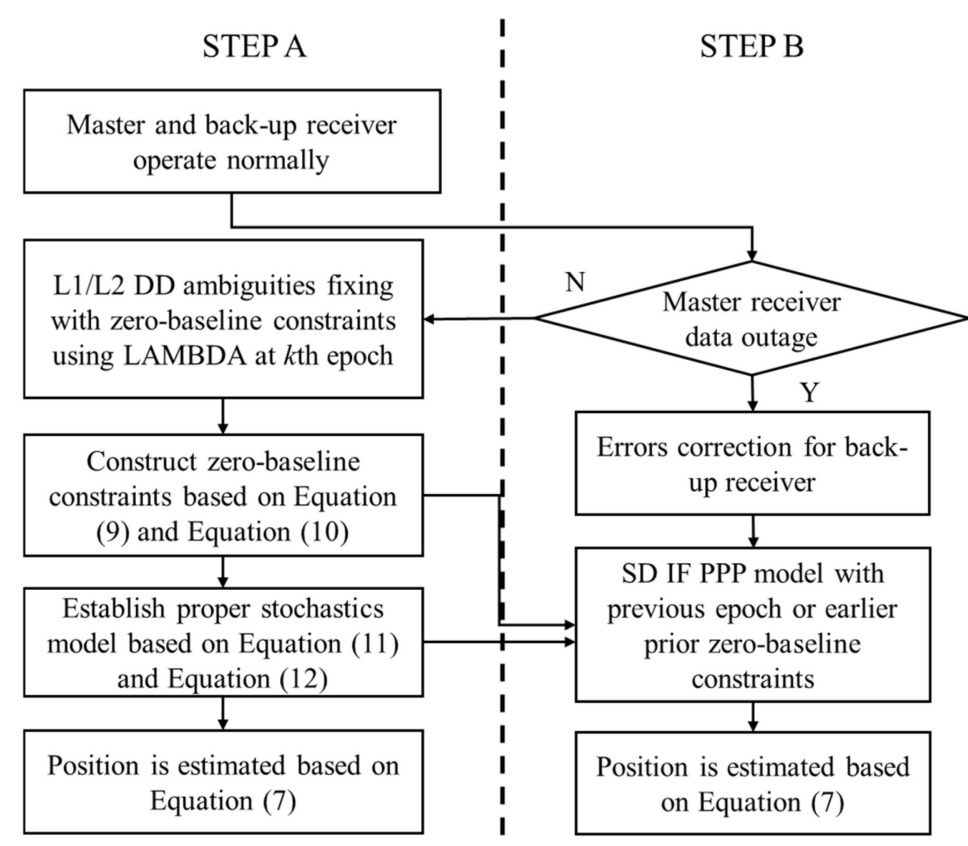

Figure 1. Flow chart of continuity enhancement method for real-time precise point positioning (PPP) with zero-baseline constraint of multi-receiver.

\section{Experiment and Discussion}

In order to evaluate continuous high-precision positioning performance of the proposed method when raw data outage occurs in a receiver, both the real-world static and kinematic GPS data were collected to sufficiently test the proposed method. The ability of instantaneous initialization is firstly verified based on the real-world static data, and the performance of continuous enhancement is evaluated based on the kinematic data. The real-time precise orbit and clock products are from the Chinese Academy of Sciences [30]. The threshold of the ratio test for the LAMBDA is set as $1 / 3$ for double-differenced ambiguities fixing. The threshold is defined as the ratio of the minimum quadratic form of residuals to the second minimum quadratic form of the residuals. The satellite elevation cutoff angle is set $10^{\circ}$.

\subsection{Static Experiment}

In this static experiment, we use zero baseline data of Curtin University with $30 \mathrm{~s}$ sampling interval from CUT0 and CUT2 in DOY 264, 2019 (saegnss2.curtin.edu/ldc/, accessed on 15 July 2017). The CUT0 and CUT2 are sharing the same antenna. The CUT0 is the master receiver, and the CUT2 is the back-up receiver. The time length for static experiment data is $24 \mathrm{~h}$. The position results can be evaluated because the coordinates of CUT0 are accurately known.

In case the master receiver has a data outage, the instantaneous initialization of backup receiver is the most important step to achieve continuity high-precision positioning enchancement of multi-receiver. Therefore, we simulated raw data outage of CUT0 every 
290 epochs by artificially introducing data outage to verify the instantaneous initialization ability of proposed method. Note that the 290 can be set to any integer and the duration of the outage is $1 \mathrm{~s}$. The left panel of Figure 2 shows the position results of CUT0 under BSSD IF PPP model. Once the simulated raw data outage of CUT0 happens, the continuous highprecision positioning performance will be lost due to re-initialization event. In contrast, the satellite signals can be continuously locked by CUT2 and the baseline between CUT0 and CUT2 is zero. The right panel of Figure 2 shows the position results of CUT2 without signal reacquisition. The results show that the continuity of high-precision positioning can be maintained after the first initialization, and the root mean square (RMS)s of position errors are $2.4 \mathrm{~cm}, 1.8 \mathrm{~cm}$, and $3.4 \mathrm{~cm}$ in the east, north, and up components, respectively.
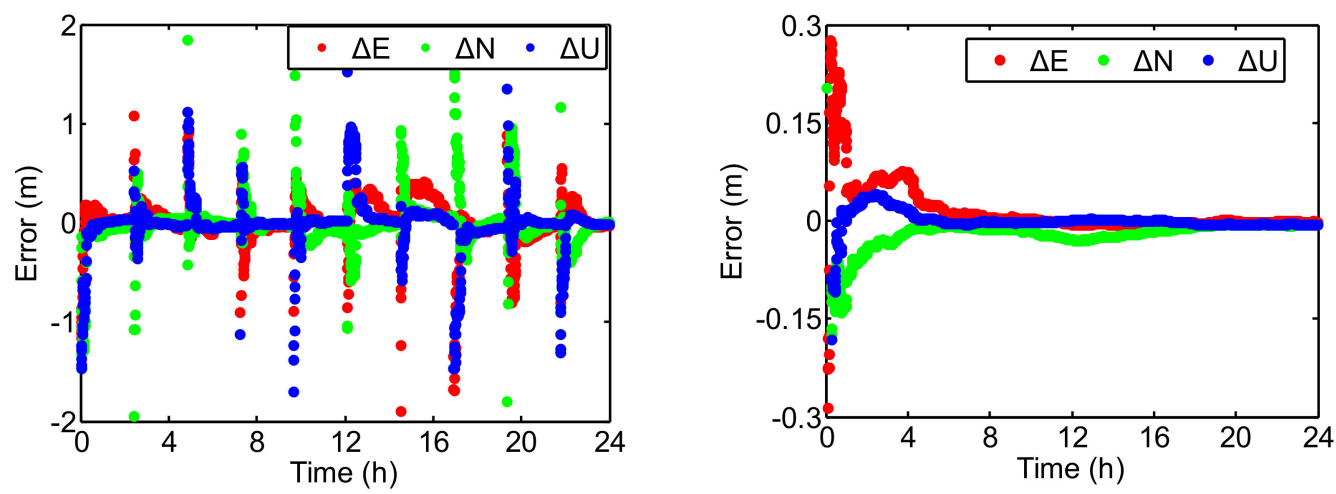

Figure 2. Position errors under single difference (SD) PPP model. The panels from (left) to (right) are CUT0 and CUT2.

In order to verify the instantaneous initialization ability of proposed method, the zero baseline constraints between CUT0 and CUT2 are first determined. The real-time kinematic (RTK) method with zero baseline constraint is adapted to determine the uncombined double differenced ambiguities of L1 and L2. The ratio test values using LAMBDA for ambiguities fixing between master and back-up receiver are given in Figure 3 . The blue point and red line denote ratio test values and threshold, respectively. It shows that all the ratio test values are much smaller than the threshold of $1 / 3$, which illustrates the reliability of double differenced ambiguities fixing with a high confidence level.

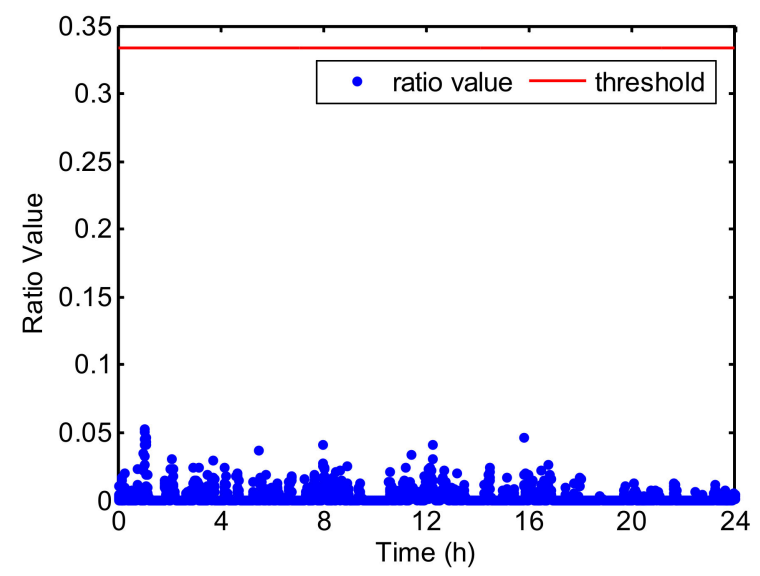

Figure 3. Ratio values of double-differenced ambiguities fixing.

The relative position between CUT0 and CUT2 can be obtained after the DD ambiguities fixing successfully. Since the baseline length is zero, the truth-value of relative position between receivers is zero, which is the perfect reference to assess the accuracy of estimated relative position. The relative position accuracy is at the order of millimeter level, as shown in Figure $4 . \Delta \mathrm{E}, \Delta \mathrm{N}$, and $\Delta \mathrm{U}$ in the caption of Figure 4 indicate position errors in the $\mathrm{E}, \mathrm{N}$, and $\mathrm{U}$ components of the ENU coordinate system. The results further verified 
the correctness and reliability of ambiguities fixing. The fixed success rate reaches $100 \%$. Therefore, the relationship of BSSD IF ambiguities between receivers can be constituted by fixing double differenced ambiguities of L1 and L2. Meanwhile, the ZWD of CUT0 is equal to CUT2 due to the zero-baseline constraint.

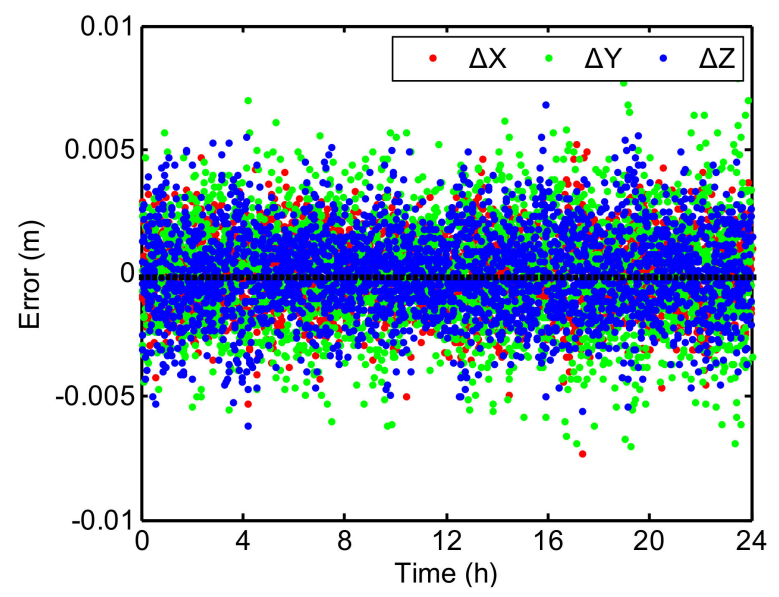

Figure 4. Relative position errors between master and back-up receiver.

Once the zero-baseline constraints are determined, the position results by the proposed method in CUT0 can be obtained, as shown in Figure 5. Compared with Figure 2, the re-initialization events disappear after the first initialization, and the position accuracy reaches centimeter level as same as CUT2. Therefore, the instantaneous initialization of RTPPP can be achieved under zero-baseline constraint of multi-receiver, and the continuous high-precision positioning performance can be maintained.

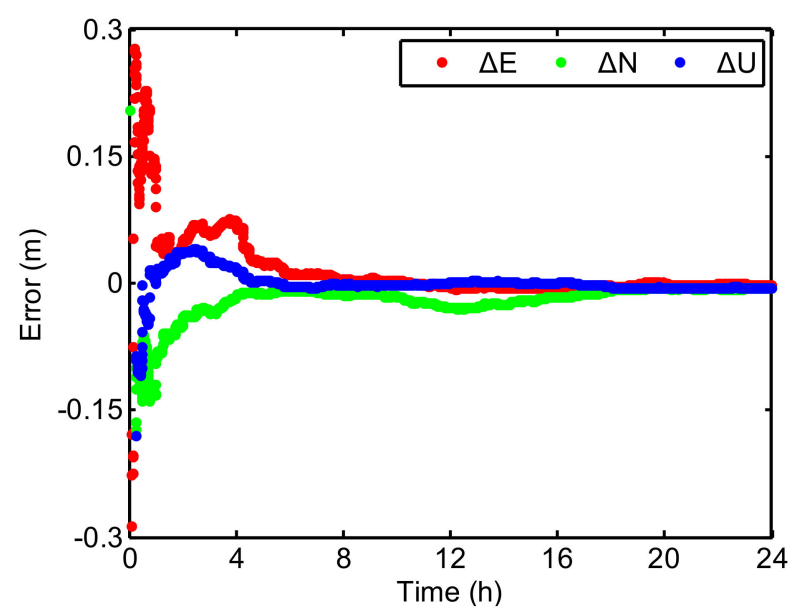

Figure 5. Position errors of multi-receiver based on zero-baseline constraint.

\subsection{Kinematic Experiment}

In order to further verify the effectiveness of proposed method in real-world applications, we use real kinematic data with $1 \mathrm{~s}$ sampling interval collected by two OEM683s via a divider and a 704 antenna on Songhua River of Harbin, China, at DOY 317, 2017. The data length is $5 \mathrm{~h}$. Figure 6 shows the trajectory of kinematic experiment, the red line represents the trajectory of the boat, and the yellow symbol Base is $3.8 \mathrm{~km}$ away from the rover. The kinematic PPP solutions were carried out to be compared by the RTK fixed solutions for further verification. Figure 7 shows the number of available satellites in multiGNSS configuration and the position dilution of precision (PDOP) during the kinematic experiment, the available satellites are more than 8 , and the PDOP is better than 3 . 


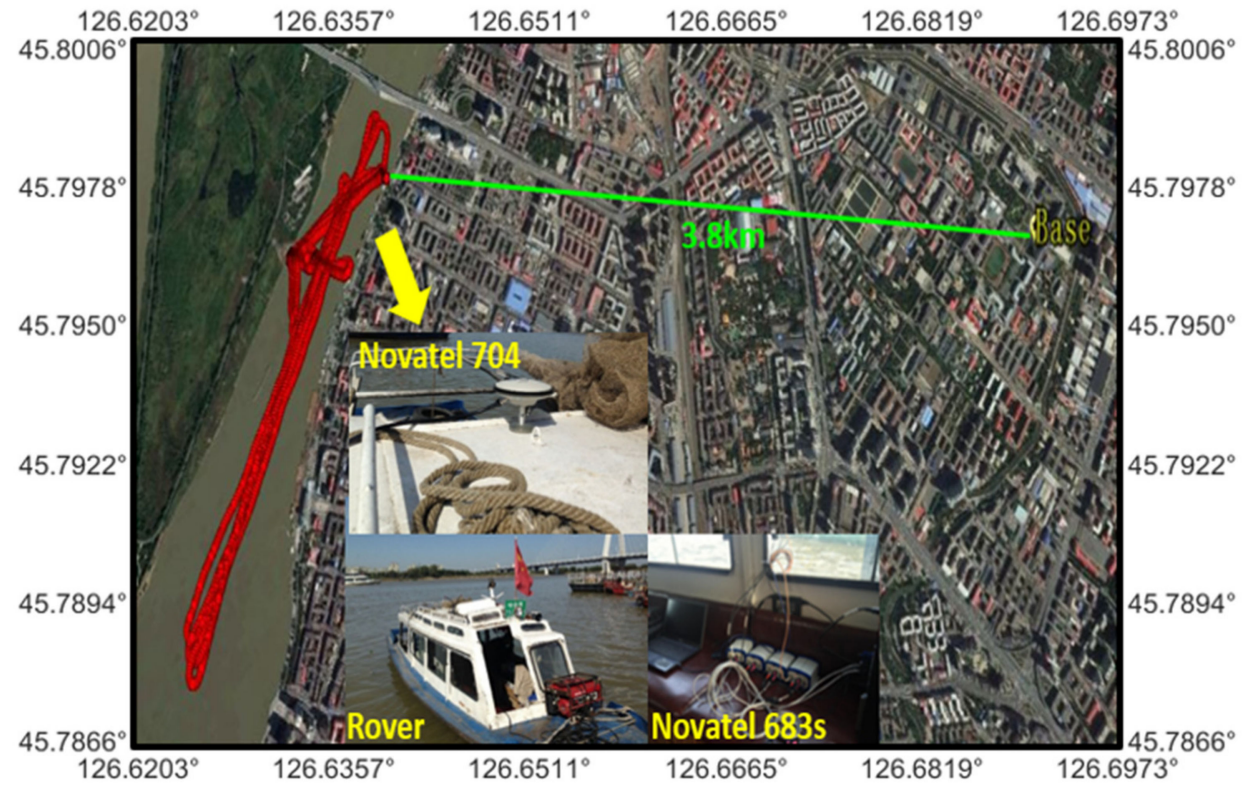

Figure 6. Kinematic experiment trajectory.

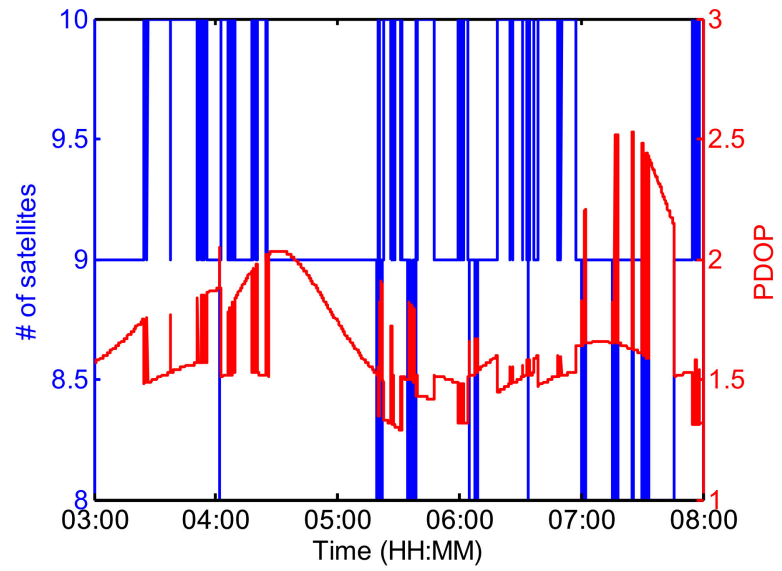

Figure 7. Available satellites and position dilution of precision (PDOP) during the kinematic test.

The artificial data outage of master receiver was introduced during the periods of 04:00:01 to 04:59:59 and 06:00:01 to 06:59:59, and the data outage of the back-up receiver appears between 03:00:00 and 03:59:59, 05:00:01 and 05:59:59, and 07:00:01 and 08:00:00. Figure 8 represents the PPP position results of master and back-up receivers. The results show that the continuity of positioning results will be lost when the receiver has a data outage. Subsequently, the re-initialization will happen when the data recovers in the master and back-up receivers. Therefore, the data outage of single receiver will destroy the continuous high-precision positioning performance.

Both master and back-up receivers can receive the raw observations data at 04:00:00, 05:00:00, 06:00:00, and 07:00:00. Therefore, the relationship of BSSD IF ambiguities and ZWD between master and back-up receiver can be determined, and their suitable stochastic models are constructed. Figure 9 shows the PPP position errors with zero-baseline constraints. The position result consists of master and back-up receiver. The results show that the continuity of high-precision positioning can be maintained after the first initialization, even if there is a single receiver data outage. The statistics related to position accuracy and continuity of master receiver, back-up receiver, and multi-receiver configuration are shown in Table 1. Compared to single receiver configuration, the positioning continuity of the multi-receiver configuration can be improved to $100 \%$, and the RMSs of position errors without re-initialization are $0.182 \mathrm{~m}, 0.101 \mathrm{~m}$, and $0.235 \mathrm{~m}$ in the east, north, and 
vertical components, respectively. In addition, the MTTR can also be improved in multireceiver strategy, because even if there is a failure in the master receiver, the continuous high-precision positiong of back-up receiver will also maintain without outage based on zero-baseline constraints.
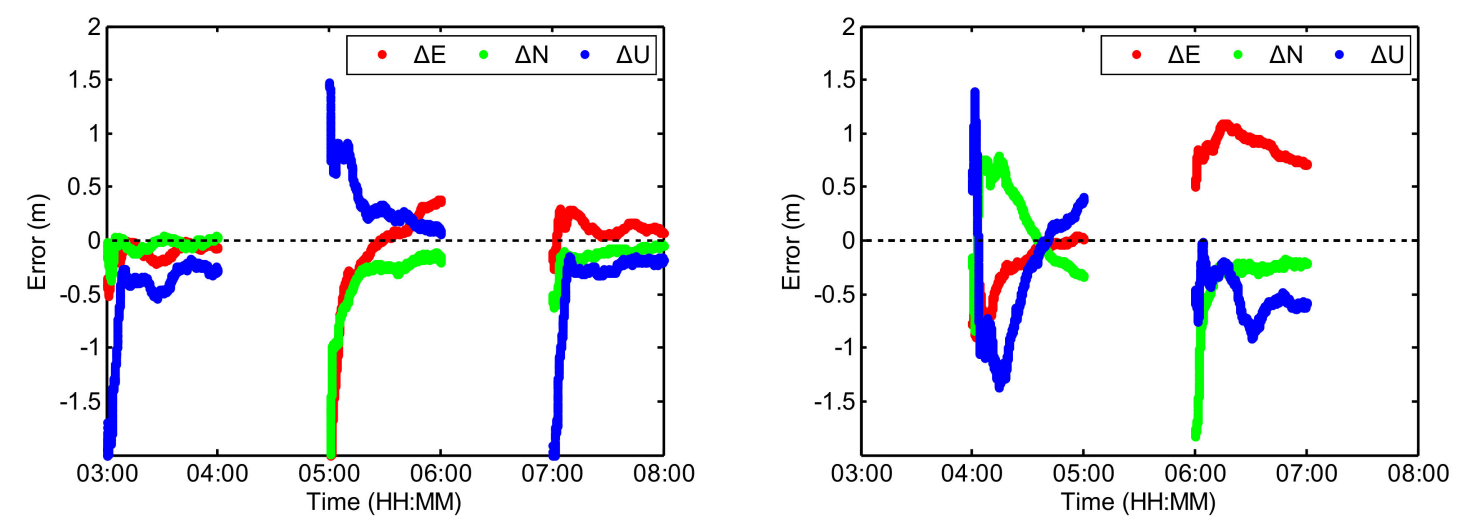

Figure 8. PPP position errors without inner-receiver constraints. The (left) panel shows the position errors of master receiver, the (right) is back-up receiver.

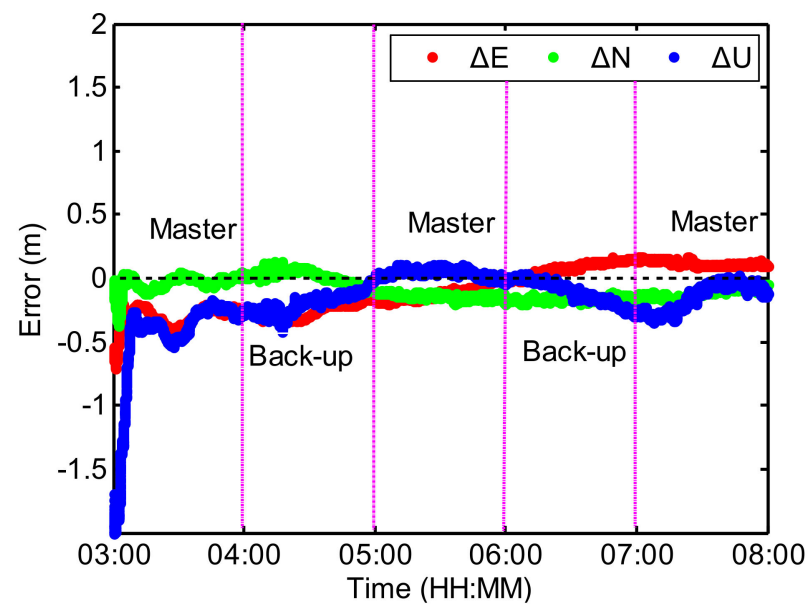

Figure 9. PPP position errors with zero-baseline constraint of multi-receiver.

Table 1. The statistics related to position accuracy and continuity of master receiver, back-up receiver, and multi-receiver configuration.

\begin{tabular}{ccccc}
\hline Configuration & $\mathbf{E}(\mathbf{m})$ & $\mathbf{N}(\mathbf{m})$ & $\mathbf{U}(\mathbf{m})$ & Continuity \\
\hline Master receiver & 0.26 & 0.222 & 0.413 & $60 \%$ \\
Back-up receiver & 0.377 & 0.268 & 0.419 & $40 \%$ \\
Multi-receivers & 0.182 & 0.101 & 0.235 & $100 \%$ \\
\hline
\end{tabular}

\section{Conclusions}

Receiver data outage is one of the reasons for continuity loss of position solutions, and the re-initialization of RT-PPP will happen when the data recover. However, the receiver data outage events are always ignored for RT applications. Hence, the purpose of this study is to achieve continuous high-precision solutions by proposing a continuity enhancement method for RT-PPP based on zero-baseline constraint of multi-receiver. Benefit from zero baseline constraints of multi-receiver, the initial BSSD IF ambiguities and ZWD can be determined instantly and reliably without baseline deformation. Moreover, in accordance with the variance-covariance propagation law, the suitable stochastic model to assign the appropriate weights for zero baseline constraints can be obtained, which is beneficial for improving the reliability of instantaneous initialization. Meanwhile, the MTTR in theory 
can be improved compared with a single receiver to maintain positioning continuity. The proposed method is tested using static and kinematic data. The static experiment has shown that the double-differenced uncombined ambiguities can be fixed with a high level of confidence with zero baseline constraint, thus, the initial BSSD IF ambiguities and ZWD can be determined instantly and reliably. Moreover, the instantaneous initialization of backup receiver can be achieved after the first initialization. Furthermore, the continuity loss and re-initialization of PPP caused by raw data outage were removed, and the continuous sub-decimeter level PPP solutions can be maintained after the first initialization in the kinematic experiment. Therefore, applying the proposed method, the high precision position solutions continuity of RT-PPP can be enhanced. In addition, the method can also easily be extended to other navigation systems such as GLONASS, Galileo, and BDS.

Author Contributions: Conceptualization, F.Y.; data curation, F.Y. and H.L.; investigation, F.Y., C.Z., and L.L.; methodology, F.Y.; software, F.Y.; supervision, L.Z.; validation, F.Y., L.Z., and L.L.; writingoriginal draft, F.Y., J.Z.; writing - review and editing, F.Y., L.L., and H.L. All authors have read and agreed to the published version of the manuscript.

Funding: This research was jointly funded by the National Natural Science Foundation of China (Nos. 61773132, 61633008, 61803115, 62003109), the National Key Research and Development Program (No. 2017YFE0131400), the 7th Generation Ultra Deep Water Drilling Unit Innovation Project sponsored by Chinese Ministry of Industry and Information Technology, the Heilongjiang Province Research Science Fund for Distinguished Young Scholars (No. JC2018019), and the Fundamental Research Funds for Central Universities (Nos. HEUCFP201768, 3072019CF0401, 3072020CFJ0402).

Data Availability Statement: The data supporting this research can be found at the hyperlinks as: http:/ / saegnss2.curtin.edu/ldc/rinex3/daily (accessed on 15 July 2017). In addition, upon a reasonable request, the observation data that support the findings of this study are available from the author (F.Y.).

Acknowledgments: The authors acknowledge the Chinese Academy of Sciences and Curtin University for providing the data.

Conflicts of Interest: The authors declare no conflict of interest.

\section{References}

1. Yang, F.; Zhao, L.; Li, L.; Feng, S.; Cheng, J. Performance evaluation of kinematic BDS/GNSS real-time precise point po-sitioning for maritime positioning. J. Navig. 2019, 72, 34-52. [CrossRef]

2. Nie, Z.; Liu, F.; Gao, Y. Real-time precise point positioning with a low-cost dual-frequency GNSS device. GPS Solut. 2020, 24, 9. [CrossRef]

3. Tu, R.; Ge, M.; Zhang, H.; Huang, G. The realization and convergence analysis of combined PPP based on raw observation. Adv. Space Res. 2013, 52, 211-221. [CrossRef]

4. Lou, Y.; Zheng, F.; Gu, S.; Wang, C.; Guo, H.; Feng, Y. Multi-GNSS precise point positioning with raw single-frequency and dual-frequency measurement models. GPS Solut. 2015, 20, 849-862. [CrossRef]

5. Li, X.; Ge, M.; Dai, X.; Ren, X.; Fritsche, M.; Wickert, J.; Schuh, H. Accuracy and reliability of multi-GNSS real-time pre-cise positioning: GPS, GLONASS, BeiDou, and Galileo. J. Geod. 2015, 89, 607-635. [CrossRef]

6. Li, P.; Zhang, X. Integrating GPS and GLONASS to accelerate convergence and initialization times of precise point posi-tioning. GPS Solut. 2014, 18, 461-471. [CrossRef]

7. Chen, X.M.; Allison, T.; Cao, W.; Ferguson, K.; Grünig, S.; Gomez, V.; Kipka, A.; Köhler, J.; Landau, H.; Leandro, R. Trimble RTX, an innovative new approach for network RTK. In Proceedings of the ION-GNSS-2011, Portland, OR, USA, 20-23 September 2011; pp. 2214-2219.

8. Ge, M.; Gendt, G.; Rothacher, M.; Shi, C.; Liu, J. Resolution of GPS carrier-phase ambiguities in Precise Point Positioning (PPP) with daily observations. J. Geod. 2008, 82, 389-399. [CrossRef]

9. Aggrey, J.; Bisnath, S. Improving GNSS PPP Convergence: The Case of Atmospheric-Constrained, Multi-GNSS PPP-AR. Sensors 2019, 19, 587. [CrossRef] [PubMed]

10. El-Mowafy, A.; Deo, M.; Kubo, N. Maintaining real-time precise point positioning during outages of orbit and clock corrections. GPS Solut. 2017, 21, 937-947. [CrossRef]

11. Nie, Z.; Gao, Y.; Wang, Z.; Ji, S.; Yang, H.; Nie, Z. An approach to GPS clock prediction for real-time PPP during outages of RTS stream. GPS Solut. 2017, 22, 14. [CrossRef]

12. Yang, F.; Zhao, L.; Li, L.; Cheng, J.; Zhang, J. Ionosphere-Constrained Triple-Frequency Cycle Slip Fixing Method for the Rapid Re-Initialization of PPP. Sensors 2018, 19, 117. [CrossRef] 
13. Luo, X.; Gu, F.; Lou, Y.; Chen, B.; Song, W. Better thresholds and weights to improve GNSS PPP under ionospheric scin-tillation activity at low latitudes. GPS Solut. 2020, 24, 17. [CrossRef]

14. Xiao, G.; Mayer, M.; Heck, B.; Sui, L.; Zeng, T.; Zhao, D. Improved time-differenced cycle slip detect and repair for GNSS undifferenced observations. GPS Solut. 2018, 22, 6. [CrossRef]

15. Zhang, X.; Li, X. Instantaneous re-initialization in real-time kinematic PPP with cycle slip fixing. GPS Solut. 2012, 16, 315-327.

16. Jacek, P. Precise GNSS single epoch positioning with multiple receiver configuration for medium-length baselines: Methodology and performance analysis. Meas. Sci. Technol. 2015, 26, 035002.

17. Song, C.; Hao, J. Instantaneous Re-convergence of Kinematic PPP by the Use of Relationship Between Multiple Receiver Ambiguity. Wuhan Daxue Xuebao 2017, 42, 595-599, 690.

18. Liu, Y.; Liu, F.; Gao, Y.; Zhao, L. Implementation and Analysis of Tightly Coupled Global Navigation Satellite System Precise Point Positioning/Inertial Navigation System (GNSS PPP/INS) with Insufficient Satellites for Land Vehicle Naviga-tion. Sensors 2018, 18, 4305. [CrossRef]

19. Song, C.; Hao, J.; Zhang, H. A Method to Accelerate PPP Re-Convergence with Prior Troposphere Delay Constraint. J. Geom. Sci. Technol. 2015, 32, 441-444.

20. Yao, Y.; Yu, C.; Hu, Y. A New Method to Accelerate PPP Convergence Time by using a Global Zenith Troposphere Delay Estimate Model. J. Navig. 2014, 67, 899-910. [CrossRef]

21. Tu, R.; Zhang, R.; Zhang, P.F.; Liu, J.; Lu, X. An approach for real-time fast point positioning of the BeiDou Navigation Satellite System using augmentation information. Meas. Sci. Technol. 2018, 29, 075003. [CrossRef]

22. Remondi, B.W. Global positioning system carrier phase: Description and use. J. Geod. 1985, 59, 361-377. [CrossRef]

23. Kouba, J. A Guide to Using International GNSS Service (IGS) Products. 2019. Available online: https:/ /igscb.jpl.nasa.gov/igscb/ resource/pubs/UsingIGSProductsVer21.pdf (accessed on 15 July 2017).

24. Petit, G.; Luzum, B. IERS_IERS Conventions (2010); IERS Technical Note No. 36; Verlag des Bundesamts für Kartographie und Geodäsie: Frankfurt am Main, Germany, 2010; ISBN 3-89888-989-6.

25. Zumberge, J.F.; Heflin, M.B.; Jefferson, D.C.; Watkins, M.M.; Webb, F.H. Precise point positioning for the efficient and robust analysis of GPS data from large networks. J. Geophys. Res. Space Phys. 1997, 102, 5005-5017. [CrossRef]

26. Guo, F.; Zhang, X.; Wang, J.; Ren, X. Modeling and assessment of triple-frequency BDS precise point positioning. J. Geod. 2016, 90, 1223-1235. [CrossRef]

27. Boehm, J.; Niell, A.; Tregoning, P.; Schuh, H. Global Mapping Function (GMF): A new empirical mapping function based on numerical weather model data. Geophys. Res. Lett. 2006, 33, 07304. [CrossRef]

28. Teunissen, P.J.G. The least-squares ambiguity decorrelation adjustment: A method for fast GPS integer ambiguity estimation. J. Geod. 1995, 70, 65-82. [CrossRef]

29. Tang, X.; Roberts, G.W.; Hancock, C.M.; Yu, J. GPS/BDS relative positioning assessment by zero baseline observation. Measurement 2018, 116, 464-472. [CrossRef]

30. Ding, W.; Tan, B.; Chen, Y.; Teferle, F.N.; Yuan, Y. Evaluation of a regional real-time precise positioning system based on GPS/BeiDou observations in Australia. Adv. Space Res. 2018, 61, 951-961. [CrossRef] 